\title{
Possible dendritic contribution to unimodal numerosity tuning and Weber-Fechner law-dependent numerical cognition
}

\author{
Kenji Morita* \\ RIKEN Brain Science Institute, Wako, Japan
}

\section{Edited by:}

Xiao-Jing Wang, Yale University School of Medicine, USA

Reviewed by:

Sridhar Raghavachari, Duke University, USA

Andreas Nieder, University of

Tuebingen, Germany

${ }^{*}$ Correspondence:

Kenji Morita, Department of Cognitive Neuroscience, Graduate School of

Medicine, The University of Tokyo,

7-3-1 Hongo, Bunkyo-ku, Tokyo

113-0033, Japan.

e-mail:kmorita@m.u-tokyo.ac.jp
Humans and animals are known to share an ability to estimate or compare the numerosity of visual stimuli, and this ability is considered to be supported by the cortical neurons that have unimodal tuning for numerosity, referred to as the numerosity detector neurons. How such unimodal numerosity tuning is shaped through plasticity mechanisms is unknown. Here, I propose a testable hypothetical mechanism based on recently revealed features of the neuronal dendrite, namely, cooperative plasticity induction and nonlinear input integration at nearby dendritic sites, on the basis of the existing proposal that individual visual stimuli are represented as similar localized activities regardless of the size or the shape in a cortical region in the dorsal visual pathway. Intriguingly, the proposed mechanism naturally explains a prominent feature of the numerosity detector neurons, namely, the broadening of the tuning curve in proportion to the preferred numerosity, which is considered to underlie the known Weber-Fechner lawdependent accuracy of numerosity estimation and comparison. The simulated tuning curves are less sharp than reality, however, and together with the evidence from human imaging studies that numerical representation is a distributed phenomenon, it may not be likely that the proposed mechanism operates by itself. Rather, the proposed mechanism might facilitate the formation of hierarchical circuitry proposed in the previous studies, which includes neurons with monotonic numerosity tuning as well as those with sharp unimodal tuning, by serving as an efficient initial condition.

Keywords: dendrite, cooperative plasticity, self-organizing map, numerosity-tuned neurons, numerical competence, Weber-Fechner law

\section{INTRODUCTION}

Humans and animals are known to share an ability to estimate or compare the numerosity of sensory stimuli or voluntary movements, referred to as the number sense (Dehaene, 1997). Difficulty of the comparison of two numerosities, measured by the response time or the error rate, is known to increase along with the decrease in their ratio (Brannon and Terrace, 1998; Dehaene and Changeux, 1993; Gallistel and Gelman, 1992) according to the Weber-Fechner law (Dehaene, 2003; Fechner, 1860; Weber, 1850). Recently, neurons showing unimodal tuning for the number of visual stimuli (Nieder and Merten, 2007; Nieder and Miller, 2004; Nieder et al., 2002) or the order of repetitive movements (Sawamura et al., 2002) were found in the monkey neocortex, and are considered to underlie such rudimentary numerical competence of animals and humans (Nieder, 2005; Nieder and Dehaene, 2009). Moreover, it was shown that the tuning curve of these neurons broadens along with the increase in the preferred numerosity (Nieder and Merten, 2007; Nieder and Miller, 2003), suggesting that the degree of interference between the tuning curves can explain the Weber-Fechner law in numerical cognition (Dehaene, 2003; Nieder and Miller, 2003).

As for the mechanism of the neuronal numerosity tuning, Dehaene and Changeux (1993) have proposed a neural network model, before the discovery of the number-selective neurons in monkeys. According to their model, each single visual stimulus (object) is represented, after being processed through the dorsal visuospatial pathway, as a similar level of localized activity in a cortical region, referred to as the location map (Figure 1A). The location map was assumed to project to another population of neurons, referred to as the summation clusters, that have monotonic numerosity tuning with various activation thresholds. Unimodal numerosity tunings then emerge in the neurons downstream of the summation clusters by receiving central excitation and lateral inhibition (Dehaene and Changeux, 1993). From a theoretical point of view, it was pointed out (Verguts and Fias, 2004) that since the mapping from the location map to the neurons with unimodal numerosity tuning is linearly inseparable, implementing this mapping requires another neural population, such as the summation clusters, in between (Minsky and Papert, 1969). In experiments, neurons having monotonic numerosity tuning have recently be found in the lateral intraparietal area (Roitman et al., 2007), in line with the prediction of the model (Dehaene and Changeux, 1993).

It is unknown, however, how such a hierarchical circuitry as proposed in the model (Dehaene and Changeux, 1993) is shaped by plasticity mechanisms in the brain. Verguts and Fias (2004) have addressed this issue, and demonstrated by simulation that if there exist neurons having monotonic numerosity tuning (i.e., the summation clusters) from the beginning, then neurons showing unimodal numerosity tuning can be developed via biologically plausible unsupervised learning. They have not shown whether or not monotonic and unimodal numerosity tuning can be developed together via unsupervised learning given the location map at the 


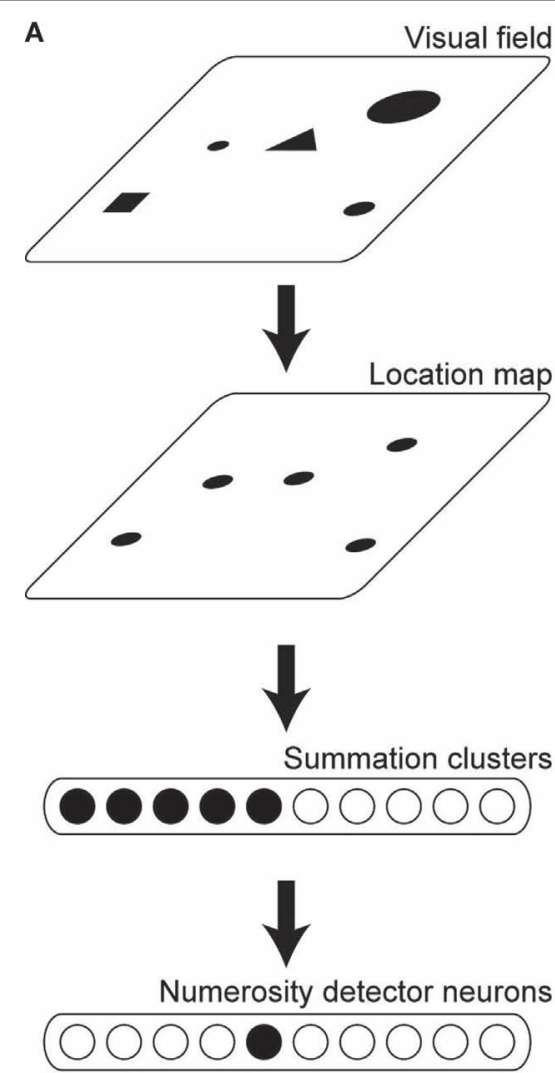

FIGURE 1 | Neural circuitry connecting to the numerosity detector neurons. (A) Hierarchical circuitry proposed in the existing models. Visual field is mapped onto the location map, where each stimulus is represented as a similar level of localized activity regardless of the original size or the shape. The location map projects to the neurons called the summation clusters, which have monotonic numerosity tuning with various activation thresholds. Unimodal

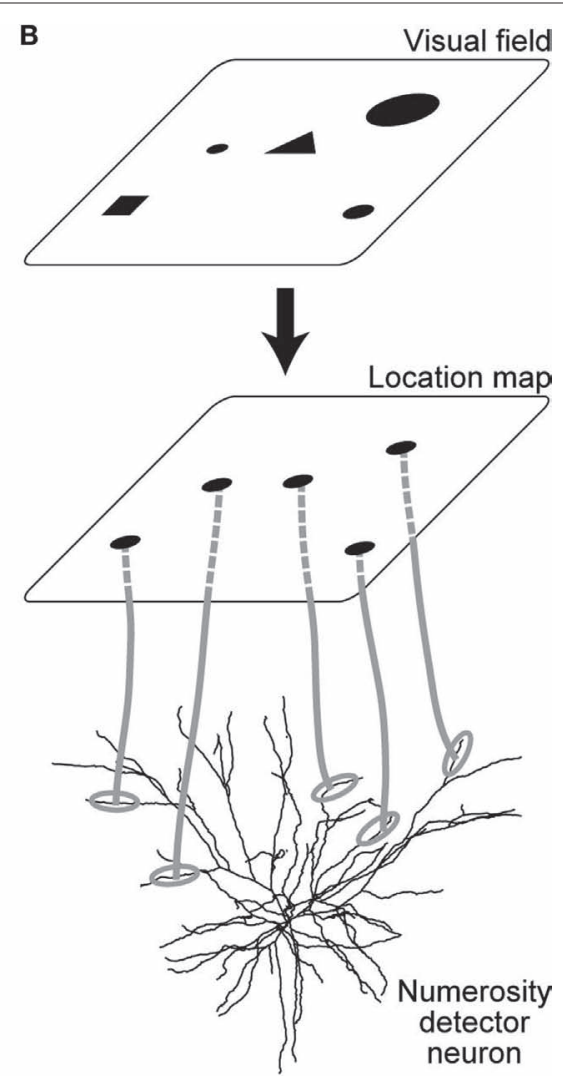

numerosity tunings emerge in the neurons downstream of the summation clusters by receiving central excitation and lateral inhibition. (B) Direct projection form the location map to the numerosity detector neurons proposed in the present study. The morphology of the neuron was adapted by permission from Macmillan Publishers Ltd: [Nature] Mainen and Sejnowski, 1996, copyright (1996) beginning. Instead, they have shown that if unimodally tuned neurons exist from the beginning, monotonically tuned neurons can be developed afterward via supervised learning. Since supervised learning is not considered to be implemented by plasticity mechanisms in the cerebral cortex, as noted by the authors, this result cannot directly relate to actual circuit formation in the cortex. Nevertheless, it reminds us of the possibility that the summation clusters might actually be developed after, rather than before, the unimodally tuned neurons are developed.

In the present paper, I propose a hypothesis on how unimodal numerosity tuning can be shaped, given the location map proposed in the previous study (Dehaene and Changeux, 1993), through cooperative plasticity induction among nearby dendritic sites (Morita, 2009), which has been theoretically predicted (Govindarajan et al., 2006; Mehta, 2004; Mel, 1993; Poirazi and Mel, 2001) and recently demonstrated in hippocampal pyramidal cells (Harvey and Svoboda, 2007; Harvey et al., 2008; Larkum and Nevian, 2008; Losonczy et al., 2008; Morita, 2009; Sjöström et al., 2008), in combination with dendritic nonlinear input integration, which was also theoretically predicted (Koch et al., 1982; Mel, 1993; Poirazi et al., 2003b) and demonstrated in hippocampal and neocortical pyramidal cells (Gasparini et al., 2004;
Liu, 2004; Losonczy and Magee, 2006; Milojkovic et al., 2005; Nevian et al., 2007; Polsky et al., 2004; Schiller et al., 2000; Wei et al., 2001). Notably, the indication of the necessity of an intermediate neural population because of the linearly inseparable nature of the mapping (Verguts and Fias, 2004) is no longer applicable given that single neurons implement multiple nonlinear operations (Poirazi et al., 2003b) (Figure 1B). According to the proposed mechanism, the tuning curve broadens roughly in proportion to the preferred numerosity, explaining the experimental results (Nieder and Merten, 2007; Nieder and Miller, 2003) and thereby providing a possible mechanistic explanation of the Weber-Fechner law in numerical cognition (Dehaene, 2003; Nieder and Miller, 2003). The simulated tuning curves are less sharp than reality, however, and together with the evidence from human imaging studies that numerical representation is a distributed phenomenon (Dehaene et al., 2004), it may not be likely that the proposed mechanism operates by itself. Rather, the proposed mechanism might initially shape rough numerosity preference, which would then facilitate the formation of the hierarchical circuitry proposed in the previous studies (Dehaene and Changeux, 1993; Verguts and Fias, 2004) by serving as an efficient initial condition. 


\section{MODEL AND SIMULATION RESULTS MODEL}

I propose a hypothetical mechanism of the formation of unimodal numerosity tuning as follows:

(I) A single visual stimulus (object) evokes a similar level of localized activity in the "location map" regardless of the size, position in the visual field, or any other physical features, and thereby the number of the localized activities on the location map matches the number of the visual stimuli (numerosity) (Figure 1B), in the same manner as considered in the previous models (Dehaene and Changeux, 1993; Verguts and Fias, 2004) (Figure 1A). In addition, the level of each localized activity decreases with the numerosity, presumably by recurrent (lateral) inhibition (Carandini and Heeger, 1994) that was demonstrated to be prevalent in neocortical circuits (Kapfer et al., 2007; Shu et al., 2003; Silberberg and Markram, 2007). Most simply, the total activity (sum) in the location map could be normalized to the same level regardless of the numerosity. I first examined this simplest case. In reality, however, whether recurrent inhibition can implement such a perfect normalization is uncertain; it may be more likely that the level of each localized activity decreases along with the numerosity but to a lesser extent so that the total activity sublinearly increases, as considered in a previous model (Verguts and Fias, 2004). Therefore, later I also examined this second possibility, showing that the main features of the model behavior remain unchanged.

(II) The original notion of self-organizing map (Amari, 1980; Kohonen, 1982; von der Malsburg, 1973) refers to that nearby inputs are mapped onto nearby neurons because of the spatially restricted recurrent excitation, combined with the Hebbian plasticity induced by concurrent pre- and post-synaptic neuronal firing activities (Figure 2A). Recently, it was suggested (Archie and Mel, 2000; Govindarajan et al., 2006; Larkum and Nevian, 2008; Mehta, 2004; Mel, 1993, 2007; Mel et al., 1998; Morita, 2009; Poirazi and Mel, 2001) that the principle of selforganizing map can also operate in a finer scale, specifically, nearby inputs can be mapped onto nearby sites on the same dendritic branch of a single neuron (Figure 2B) because of the branch-specific (i) electrical and (ii) biochemical cooperativity, possibly combined with (iii) the dendritically-regulated plasticity induced by concurrent pre-synaptic neuronal firing and post-synaptic dendritic spike generation. Here, the electrical cooperativity (i) refers to supralinear synaptic integration and dendritic spike generation, as predicted (Mel, 1993; Poirazi et al., 2003a,b) and observed in the neocortex and the hippocampus (Gasparini et al., 2004; Losonczy and Magee, 2006; Milojkovic et al., 2005; Nevian et al., 2007; Polsky et al., 2004; Schiller et al., 2000; Wei et al., 2001) while the biochemical cooperativity (ii) means spatially restricted availability of the plasticity-related proteins, as recently suggested (Govindarajan et al., 2006) and observed for Ras in the hippocampus (Harvey et al., 2008), and the plasticity induction by dendritic spikes (iii) was observed in the hippocampus (Golding et al., 2002; Harvey and Svoboda, 2007). This mechanism, referred to as the clustered plasticity (Govindarajan et al., 2006; Harvey and Svoboda, 2007), was recently demonstrated in the hippocampus (Harvey and Svoboda, 2007). Moreover, other recent findings, specifically, branch-specific change in the excitability via activity-dependent regulation of potassium channels, named the branch strength potentiation (Losonczy et al., 2008) and activity-dependent secretion of brain-derived neurotrophic factor (BDNF) from individual spines (Tanaka et al., 2008), may also facilitate such a self-organizing map at the single neuron level (Morita, 2009). The exact meaning of the "nearby inputs" in the above would depend on the nature of the inputs such as the level of the firing rate or the firing patterns, but since the relevant branch-specific events have rather long time scales, specifically, more than tens of milliseconds for the dendritic NMDA spikes (Nevian et al., 2007; Polsky et al., 2004; Rhodes, 2006; Schiller et al., 2000) and minutes or more for the local availability of plasticity-related proteins (Govindarajan et al., 2006; Harvey and Svoboda, 2007; Harvey et al., 2008), millisecond-order spike synchronization may not be necessary; being coactive in the same epoch of the order of seconds or more may be enough at least under certain conditions. Based on these considerations, I assumed that inputs from the same portion of the location map are "nearby" (in the above sense) whereas those from different portions are not, so that inputs from the same portion of the location map tend to be clustered onto single dendritic branches whereas those from different portions tend to project to different parts of the dendritic tree, or more specifically, to different dendritic branches (Figure 1B). In consequence, each localized activity on the location map, corresponding to an individual visual stimulus, usually activates a different dendritic branch, though strict one-to-one correspondence can be somewhat degraded (see below).

(III) Each dendritic branch implements nonlinear input integration through dendritic spike generation (Gasparini et al., 2004; Losonczy and Magee, 2006; Mel, 1993; Milojkovic et al., 2005; Nevian et al., 2007; Poirazi et al., 2003b; Polsky et al., 2004; Schiller et al., 2000; Wei et al., 2001). Specifically, when and only when the input to a branch exceeds a certain threshold, the branch gives an excitation to the cell body so as to contribute to action potential generation (Figures $3 \mathrm{~A}, \mathbf{B}$, small graphs in the left). I assumed that the level of the threshold varies from neuron to neuron, similar to what was assumed in the previous model (Dehaene and Changeux, 1993) but here for the dendritic threshold rather than for the somatic threshold. Threshold should also vary from branch to branch within a single neuron; this was incorporated later. The inter-neuronal variability in the threshold is expected to emerge from the difference in global properties of the neuron such as the cell size or the expression level of relevant genes, or in the amount of inhibition provided by nearby interneurons, whereas the intra-neuronal variability would emerge from morphological and physiological variations between branches. I assumed that the branch is saturated whenever it receives suprathreshold input (i.e., binary) for the sake of simplicity. This can actually be a good approximation (c.f., Nevian et al., 2007; Wei et al., 2001), although considering continuous nonlinearity (e.g., sigmoidal) might be more precise. 


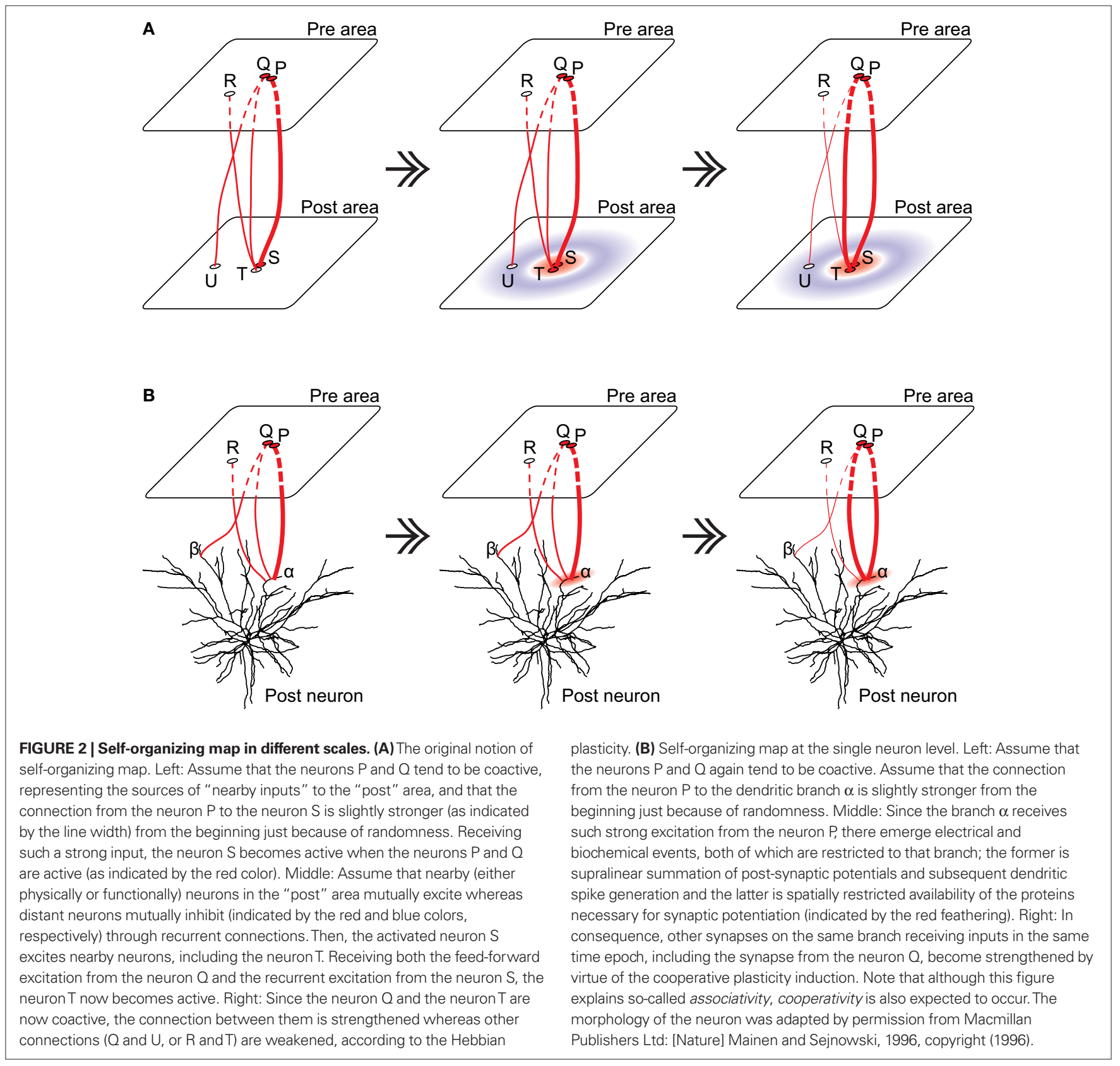

\section{SIMULATION RESULTS}

Let us see how the model works in a simple case where the neuron has four dendritic branches (Figure 3). When there are two visual stimuli, and thus two localized activities in the location map, the neuron receives inputs on two different branches (Figure 3A, second from the top). Given that these inputs are larger than the dendritic threshold, as indicated in the left small graphs, the neural activity becomes " 2 ". If there is only one visual stimulus instead, and thus input is applied only to a single branch (Figure 3A, top), the neural activity decreases to "1", because the branch is saturated and thus it cannot provide more excitation to the cell body than the previous case. Hereby this neuron turned out to be more activated, or more selective, to the numerosity 2 than to 1 , as indicated by the color in the figures. If there are three stimuli and the inputs are applied onto three branches (Figure $3 \mathrm{~A}$, third from the top), the neural activity increases to " 3 ". However, if there are four stimuli (Figure 3A, bottom), the input to individual branches can no longer exceed the threshold, because of the presumed decrease in the level of each localized activity in the location map along with the numerosity [in the assumption (I)], so that the neural activity becomes " 0 ". In this way, the neural activity differs according to the number of visual stimuli, resulting in the unimodal tuning property (Figure 3C, top). Now, consider another neuron that has a higher threshold (Figure 3B). For this neuron, a single visual stimulus evokes suprathreshold input on a single branch (Figure 3B, top) whereas two visual stimuli evoke only subthreshold inputs on two branches (Figure 3B, bottom). Therefore, the neural activity becomes " 1 " for the numerosity 1 but " 0 " for 2 (Figure 3C, bottom), 
A
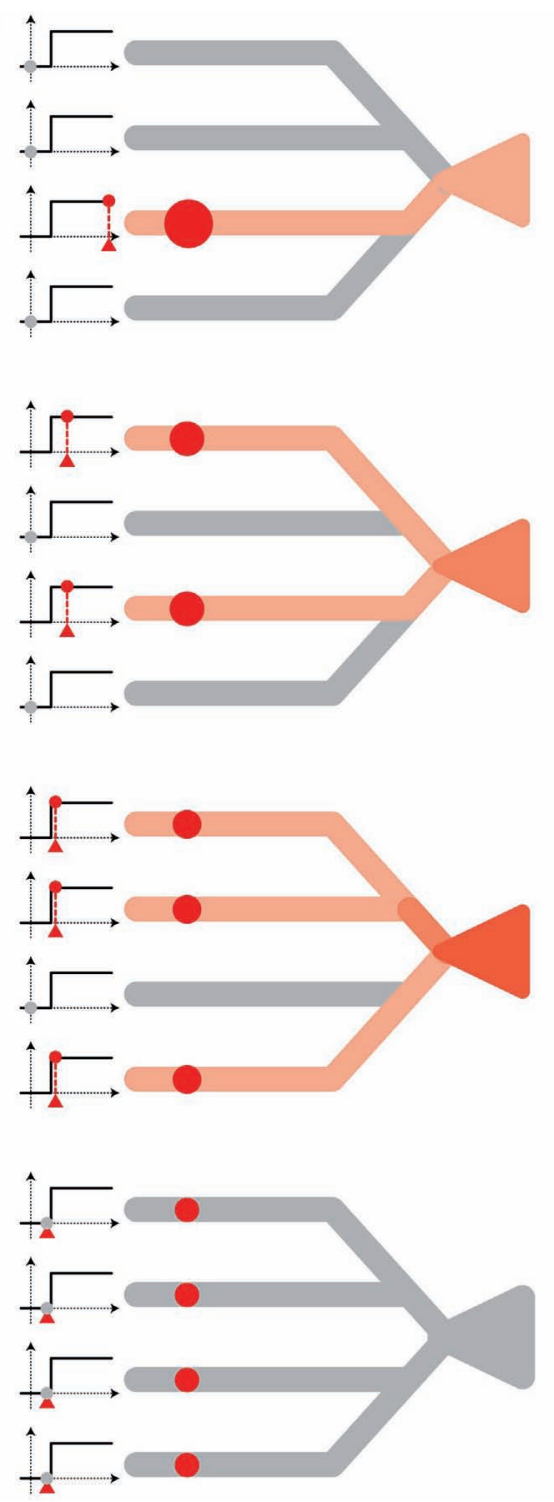

FIGURE 3 | Schematic diagram of the proposed mechanism of unimodal numerosity tuning. Consider neurons having four dendritic branches (for the purpose of explanation). (A) A neuron receives inputs (red circles) on 1, 2, 3, or 4 branches (top to bottom, respectively). In the first three cases, the input exceeds the threshold in individual branches, as shown in the left graphs, so that the neural activity increases along with the number of the branches that receive
B
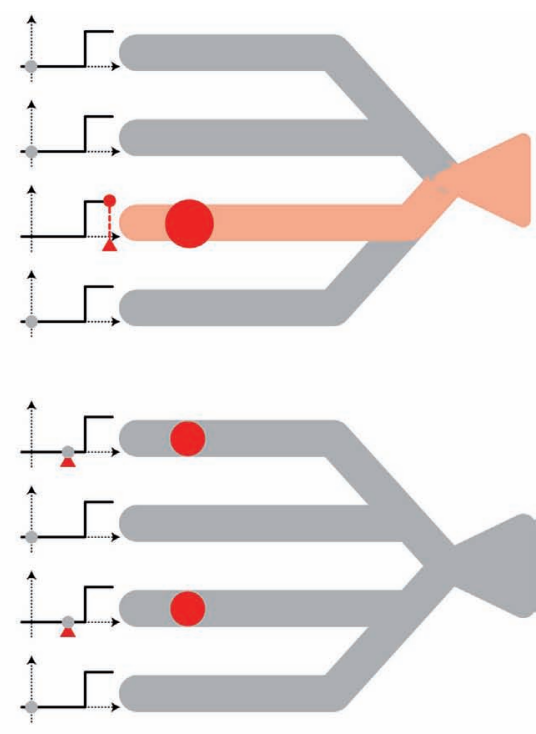

C
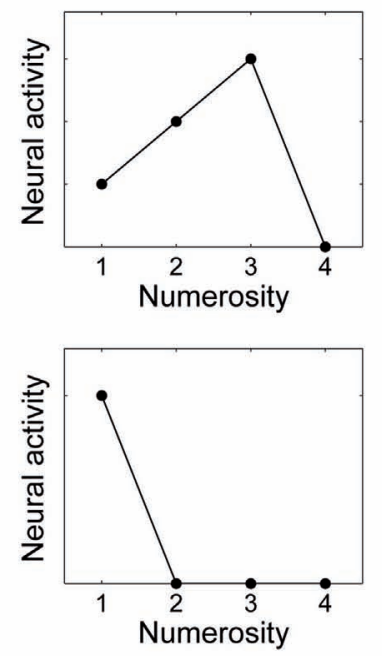

inputs (indicated by the contrast of the red color). In the last case (bottom), however, the input on each branch is below the threshold and thus the neuron remains inactive. (B) The same as (A) except that this neuron has a higher threshold. (C) Numerosity tuning of the neuron (A) (top) and the neuron (B) (bottom). The tuning is unimodal, and the preferred numerosity differs according to the level of the threshold. demonstrating that the numerosity preference differs according to the level of the threshold.

Numerosity tuning curves of three neurons, each of which has 50 dendritic branches but with three different levels of threshold, are shown in Figure 4A; neural activity is normalized so that the maximum is equal to 1 (throughout the paper), primarily for the purpose of illustration but such a normalization might actually be realized at least to some extent by homeostatic mechanisms (Ibata et al., 2008; Turrigiano and Nelson, 2004). As shown in the figure, as the threshold decreases (from blue to green, and then to red), the preferred numerosity increases. Notably, the width of the tuning curve also increases proportionally, as naturally expected from the mechanism. This is in line with the experimental observations (Nieder and Merten, 2007; Nieder and Miller, 2003, 2004; Nieder et al., 2002). More specifically, such a proportionality indicates that the widths of the tuning curves will become equal if they are plotted on the logarithmic scale (Figure 4C). Therefore, this can naturally explain the Weber-Fechner law-dependent accuracy in numerosity estimation and comparison tasks (Dehaene, 2003; Nieder and Miller, 2003). The shape of the tuning curve, however, looks different from reality; the right slope is too steep. This point can be resolved in the course of making the model more realistic. 


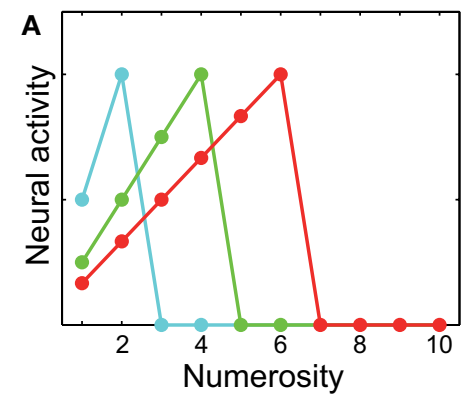

C
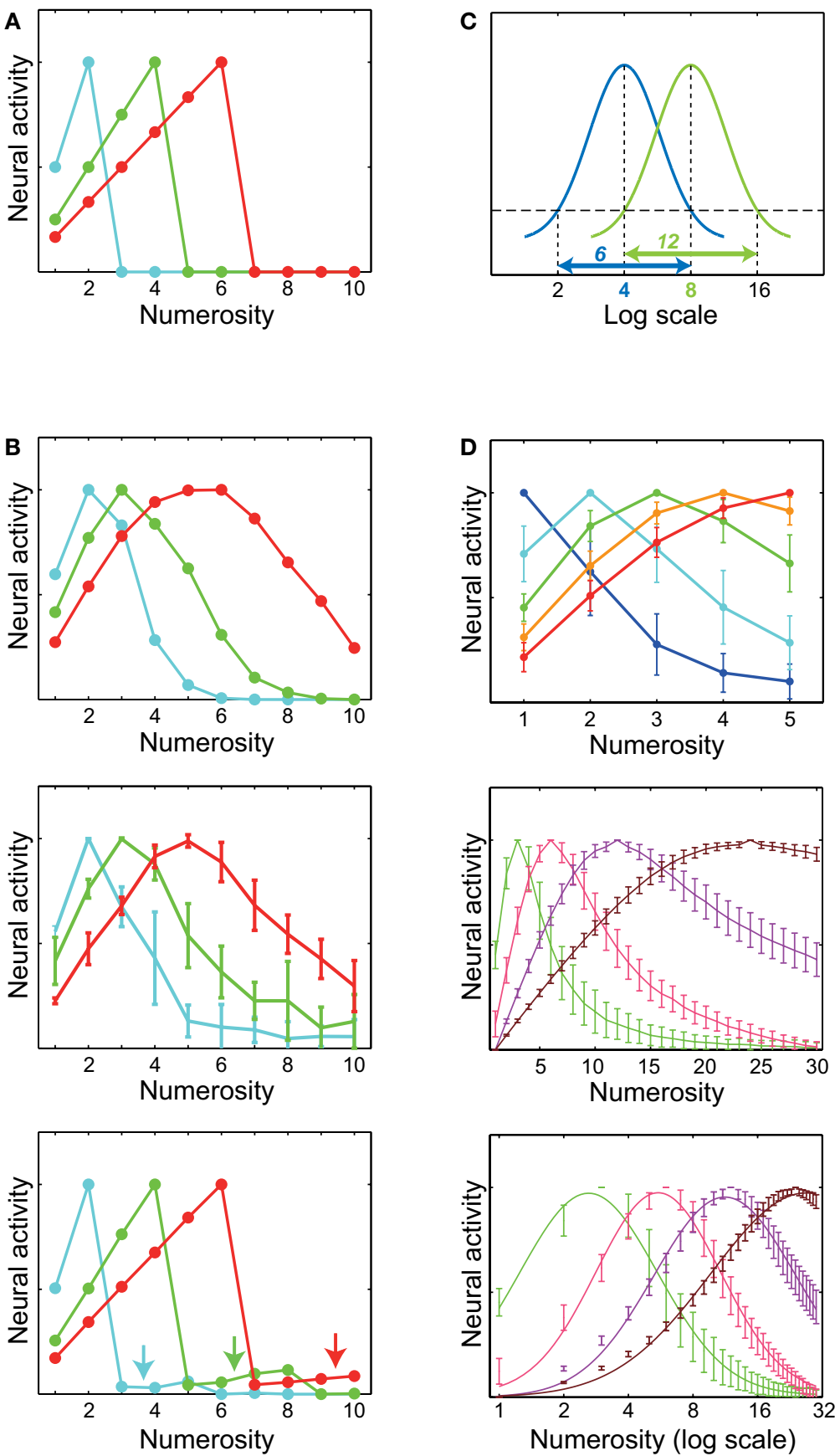
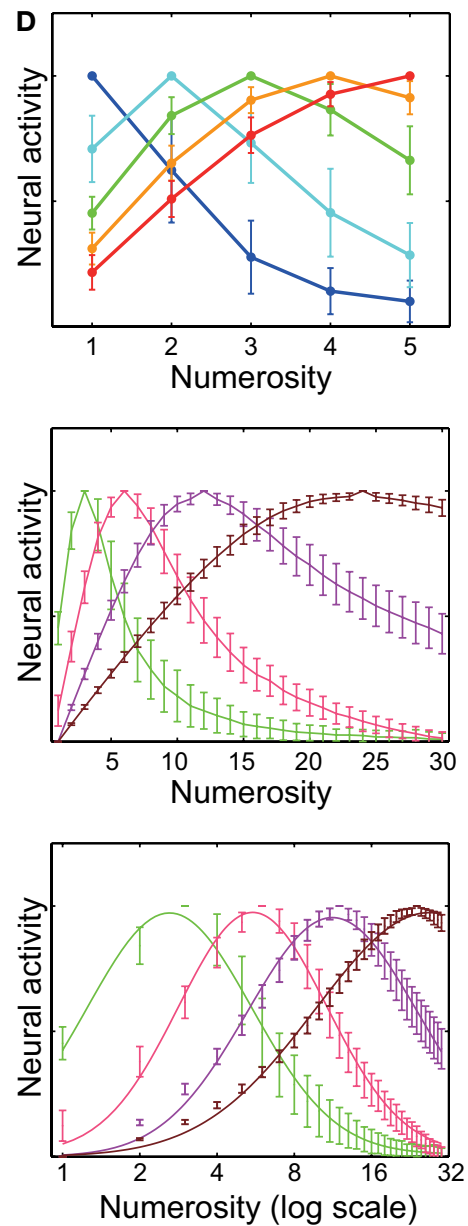

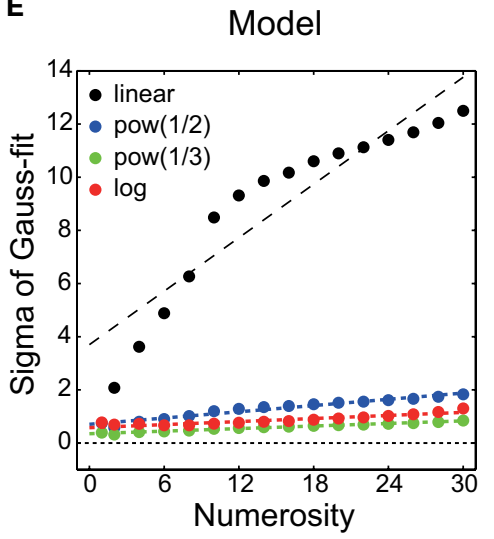

Experiment

Reprinted with permission from: Nieder \& Merten, J Neurosci, (2007) 27:5986; Fig. 7B
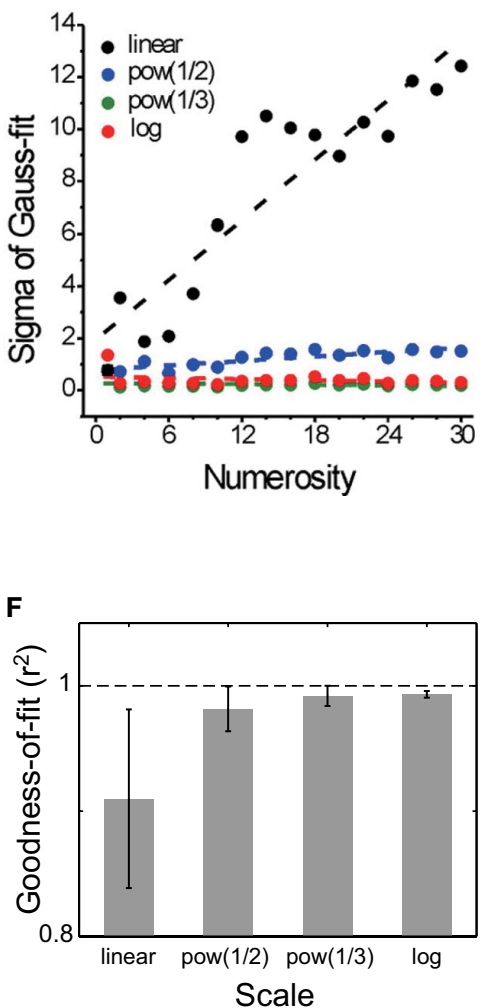

FIGURE 4 | Numerosity tuning curves obtained by simulations of the proposed mechanism. Neurons having 50 dendritic branches were considered. (A) Tuning curves of neurons with three different threshold levels are shown. As the threshold decreases (from blue to green, and then to red), the preferred numerosity increases, and notably, the width of the tuning curve also increases proportionally. (B) Possible elaborations of the model by which the tuning curve entails right-oriented asymmetry as observed in the experiments. Top: Variability in the input magnitude was incorporated. Middle: Intra-neuronal variability in the threshold was incorporated. The error bars indicate the standard deviations over ten neurons. Bottom: One-to-one correspondence between the localized activity in the location map and the dendritic branch (Figure 1B) was degraded so that each branch received inputs from up to three distant portions of the location map. (C) Schematic diagram showing that if the tuning curve has the same shape in the logarithmic scale, the peak (most preferred numerosity) and the width of the tuning curve varies proportionally. (D) Top and middle: Numerosity tunings, taking all of (B) into account, in the cases of the preferred numerosity around $1 \sim 5$ (top) or 3, 6, 12 and 24 (middle). Bottom: The same tuning curves in the middle panel plotted on the logarithmic scale, with the Gaussian fitting curves. The curves are asymmetric in the linear scale (middle), but become more symmetric in the logarithmic scale (bottom). (E) Top: How the dependence of the tuning width (the standard deviation of the Gaussian fittings) on the preferred numerosity changes with the scale. The width drastically increases with the preferred numerosity when plotted on the linear scale (black dots), whereas the change in the width is much less in the cases with the nonlinear scales (blue, red, and green dots), consistent with the experimental observations (Bottom: Reprinted from (Nieder and Merten, 2007), Figure 7B by permission of the Journal of Neuroscience.). (F) The goodness of the Gaussian fit in the different scales. The logarithmic scale gives the best fit, indicating that the tuning curve becomes most symmetric in that scale. 
First, although the input magnitude and the dendritic threshold were so far assumed to be the same for all the branches in a single neuron, in reality they are expected to differ from branch to branch at least to some extent. If such intra-neuronal branch-to-branch variability in either the input magnitude or the dendritic threshold is incorporated (modeled by Gaussian pseudo random numbers: see Model details for details) in addition to the inter-neuronal variation that was already considered, there appears a tail on the right side of the tuning curve (Figure 4B, top and middle panels, respectively). This can be explained as follows. Provided there exists variability in the level of input to branches (and no intra-neuronal variability in the threshold, for simplicity) (Figure 4B, top), then even if the average of the input (over trials and branches) is smaller than the threshold (i.e., there are more items than the preferred numerosity of the neuron of the case without the variability), input can sometimes (i.e., in some trials and for some branches) exceed the threshold by virtue of the variability, thereby causing neuronal firing. Likewise, provided there exists intra-neuronal variability in the dendritic threshold (and no variability in the input magnitude, for simplicity) (Figure 4B, middle), then even if the input is smaller than the average of the threshold (i.e., again, there are more items than the preferred numerosity of the neuron of the case without the variability), neuron can still fire when the inputs are applied onto branches whose thresholds are smaller than the average. Second, since the number of the branches is limited and the plasticity rule would not always operate perfectly, inputs from different portions on the location map might sometimes converge onto the same dendritic branch, as mentioned at the end of the assumption (II). Incorporating such an input convergence also causes an increase in the expected neural activity for larger numerosities (Figure 4B, bottom: arrows), thereby contributing to the right-side tail when it is combined with the variability introduced above. Inversely, single localized activity may be formed at an arbitrary location and thus may sometimes project to two or more branches, possibly further broadening the tuning curve, although it is possible that localized activity can only be formed at numerous but finite predetermined positions (i.e., attractors; c.f. Brody et al., 2003; Wang, 2001), each of which projects to a single dendritic branch.

The top and middle panels of Figure 4D show the numerosity tunings, taking into account all of the above, namely, variability in the input (Figure 4B, top) as well as in the threshold (Figure 4B, middle) and the input convergence (Figure $4 \mathbf{B}$, bottom), in the cases of the preferred numerosity at 15 (top) or $3,6,12$, and 24 (middle). They appear comparable to those observed in the experiments (Nieder and Merten, 2007; Nieder and Miller, 2003, 2004; Nieder et al., 2002); specifically, the model well reproduced the observation that the width increases with the preferred numerosity. The bottom panel of Figure 4D shows the same tuning curves in the middle panel plotted on the logarithmic scale, with the fitting curves of the normal distributions (Gaussians). As shown in the panel, the tuning widths become more comparable and the shapes become more symmetric in the log scale than in the linear scale (middle panel), in line with the experiments (Nieder and Merten, 2007; Nieder and Miller, 2003). The top panel of Figure 4E shows how the dependence of the tuning width, defined by the standard deviation of the fitted Gaussian functions, on the preferred numerosity changes with the scale on which the tuning curves are plotted. As shown in the panel, the width drastically increases with the preferred numerosity when plotted on the linear scale (black dots), whereas the changes in the width are much less in the cases with the nonlinear scales (blue, green, or red dots), consistent with the experimental observations shown in the bottom panel (Reprinted from (Nieder and Merten, 2007), Figure 7B by permission of the Journal of Neuroscience). Figure $4 \mathrm{~F}$ shows the goodness of the Gaussian fit in the different scales; the nonlinear scales give better fits than the linear scale, reflecting that the tuning curves are more symmetric in the nonlinear scales (Figure 4D, middle and bottom).

\section{ROBUSTNESS AND PLAUSIBILITY}

Next, I examined how the behavior of the model depends on the parameter values, in particular, the degree of variability in the input magnitude (Figure 4B, top) and the dendritic threshold (Figure 4B, middle), or the number of dendritic branches. Figure $5 \mathrm{~A}$ shows the simulation results when the variability in the input magnitude and the dendritic threshold was decreased; standard deviation per mean was 0.2 for both, compared with 0.3 in the simulations described in the above (Figures 4D-F). As shown in Figure 5A, emergence of the unimodal number selectivity and broadening of the tuning curves along with the numerosity were preserved, whereas the shape of the tuning curve was affected, i.e., the right tail was reduced (decayed faster), as can be expected from the mechanism mentioned before (Figure 4B, top and middle). Consequently, the tuning curves became better fitted by Gaussian in the scale of the power functions rather than in the log scale, although the log scale still gives better Gaussian fitting (i.e., better symmetry) than the linear scale (Figure 5Ae). Similar outcomes were obtained when the convergence of the multiple inputs corresponding to different portions of the location map onto the same branch (Figure 4B, bottom), which was assumed (up to three branches) in the simulations described in the above (Figures 4D-F), was not considered, as shown in Figure 5B. Conversely, increasing the variability in the input magnitude and the dendritic threshold (standard deviation per mean was 0.4 , compared with the original 0.3 ), as well as increasing the degree of input convergence (i.e., allowing the convergence of inputs from up to five different portions, compared with the original up to three portions), thickened the right tail of the tuning curves so that the logarithmic scaling gave even better symmetry than the scaling with the power functions (Figures 5C,D, respectively), compared with the results with the original parameter values (Figures 4D-F), though the difference between Figures 4 and $5 \mathrm{D}$ is small. It is therefore suggested that the model can well reproduce the experimental observation that the tuning curve becomes most symmetric in the logarithmic scale (Nieder and Merten, 2007; Nieder and Miller, 2003), provided there exist sufficient variability in the input magnitude and the dendritic threshold and/or a certain degree of input convergence. Figures 5E,F show the results of the cases where the number of dendritic branches was decreased (30 branches, compared with the original 50 branches) or increased (100 branches), respectively. The variability in the input magnitude and the dendritic threshold was also increased in the latter case (standard deviation per mean was 0.4 , compared with the original 0.3). As shown in the figures, the main features of the model behavior explained above (Figures 4D-F) were preserved 
A Variability decreased

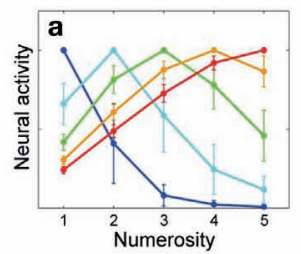

B Input convergence removed
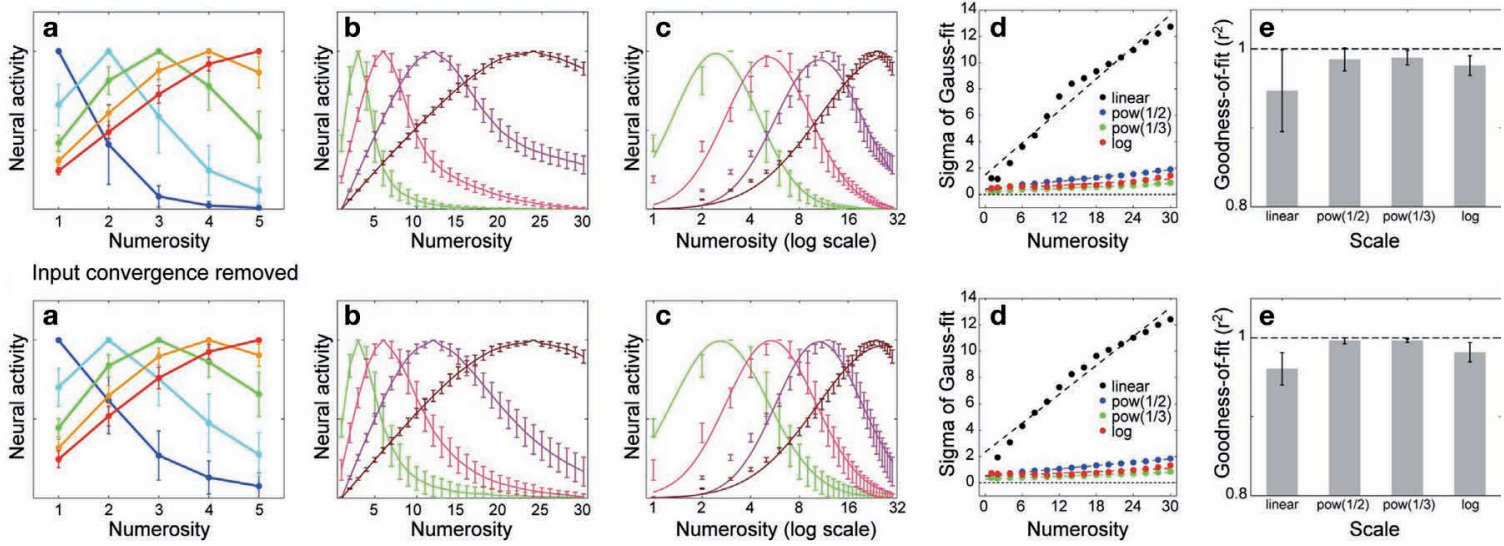

C
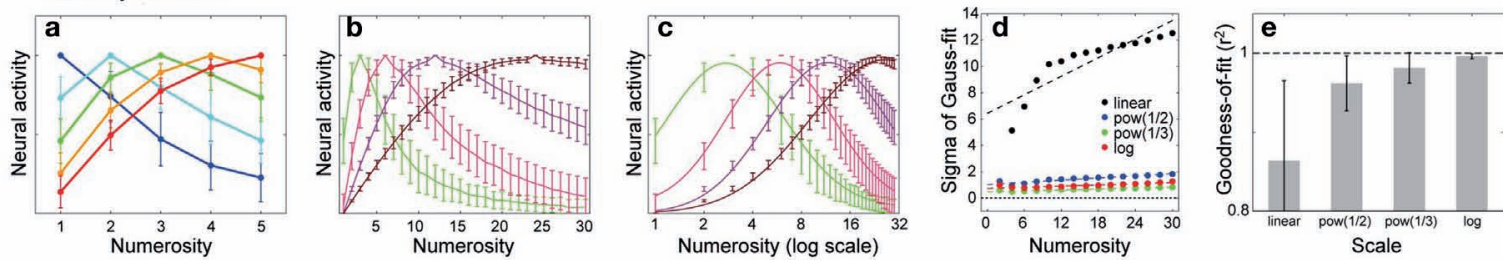

D Input convergence increased
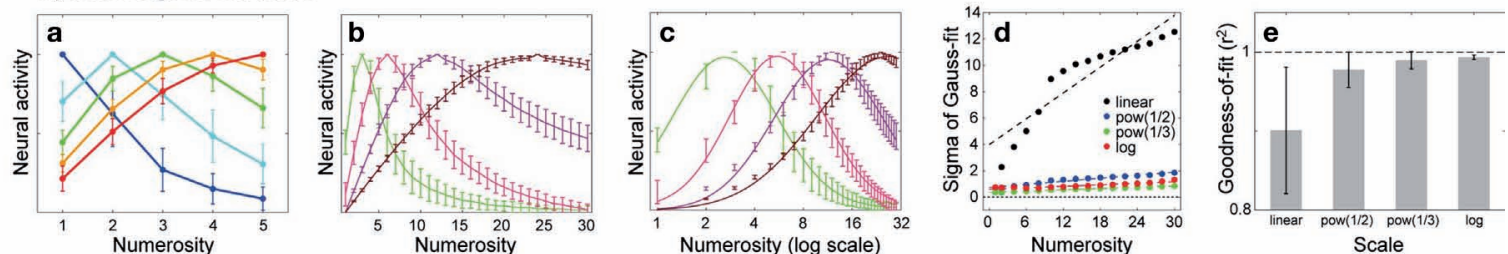

E Branches decreased
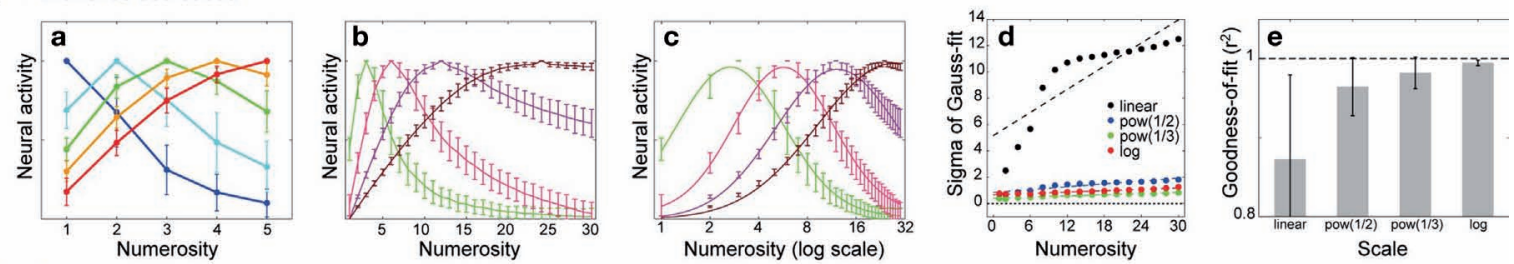

F Banches increased
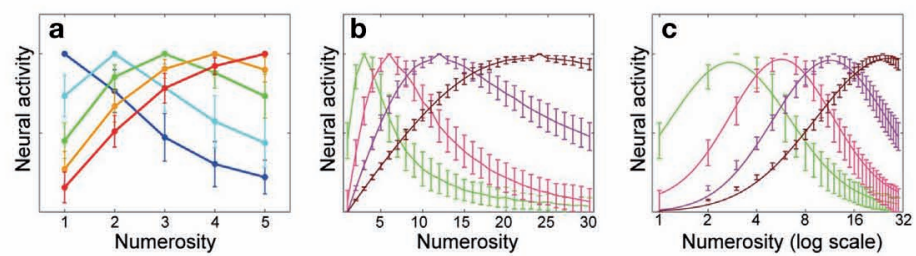

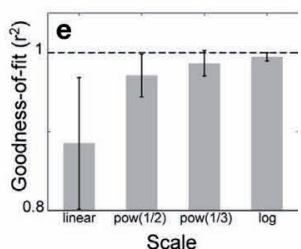

FIGURE $\mathbf{5}$ | Determinants of the tuning curve shape. $(\mathbf{A}, \mathbf{C})$ Simulation results when the variability in the input magnitude and in the dendritic threshold was decreased [(A); standard deviation per mean 0.2 for both, compared with the original 0.3 in Figures 4D-F] or increased [(C); 0.4$]$. As the variability was decreased or increased, the right tail of the tuning curve was reduced or thickened [most apparent in (b)], respectively, as can be expected from the mechanism mentioned before (Figure 4B, top and middle). (B,D) Results when the degree of convergence of the inputs from different portions of the location map was decreased [(B); each branch receives input only from a single portion] or increased [(D); each branch receives input from up to 5, compared with the original 3 in Figures 4D-F, different portions]. As the input convergence was removed or increased, the right tail of the tuning curve was reduced or thickened, respectively, as can be expected from the mechanism mentioned before (Figure $\mathbf{4 B}$, bottom). From these results (A-D), it is suggested that the model can well reproduce the experimental observation that the tuning curve becomes most symmetric in the logarithmic scale, provided there exist sufficient variability in the input magnitude and the dendritic threshold and/or a certain degree of input convergence. $(\mathbf{E}, \mathbf{F})$ Results when the number of dendritic branches was decreased [(E); 30 branches, compared with the original 50 branches in Figures 4D-F] or increased [(F); 100 branches]. The variability in the input magnitude and the dendritic threshold was the same as the original $(0.3)$ in $(\mathbf{E})$, and increased (0.4) in (F). The degree of input convergence was assumed to the same as the original (up to three different portions of the location map). The main features of the model behavior were preserved in those cases. $(\mathbf{a}, \mathbf{b})$ Numerosity tunings in the cases of the preferred numerosity around $1 \sim 5$ (a) or 3, 6, 12, and 24 (b). (c) The same tuning curves in (b) were plotted on the logarithmic scale, with the Gaussian fitting curves. (d) Dependence of the tuning width (the standard deviation of the Gaussian fittings) on the preferred numerosity in four different scales. (e) Goodness of the Gaussian fit in the different scales. 
in those cases [if the standard deviation per mean was set to 0.3 in the case of 100 branches, the power function scale gave slightly better Gaussian fits than the log scale (not shown)].

In the simulations so far presented, it was assumed that the summation of the localized activities in the location map, or equivalently, the summation of the inputs to all the dendritic branches of individual numerosity detector neurons, was normalized to be around a constant level (except for the presumed input variability) regardless of the numerosity of visual stimuli. Such a normalization of the linear summation, however, may not be the case in reality, as mentioned before [in the assumption (I)]. Therefore, I also examined the case in which the level of each localized activity, or input to each branch, again decreases with the numerosity but to a lesser extent than the previous case so that the total activity in the location map (i.e., the linear summation of the inputs to the branches) sublinearly increases with the numerosity. Specifically, I examined, as an example, the case where the sum of the squares of the localized activities is assumed to be constant (except for the variability), which is the same assumption as considered in a previous network-based model (Verguts and Fias, 2004). Figure 6 shows the results of the simulations, in which the variability (standard deviation per mean) in the input magnitude and the dendritic threshold (Figure 4B, top and middle) was set to 0.25 and the convergence of multiple inputs corresponding to different portions of the location map onto the same branch (Figure 4B, bottom) was not considered [as originally assumed in the assumption (II)]. As shown in Figure 6, the main features of the model behavior, namely, formation of the unimodal numerosity selectivity, broadening of the tuning curve roughly in proportion to the preferred numerosity (explaining the WeberFechner law) better symmetry in the nonlinear scales than in the linear scale, and better symmetry in the log scale than in the scales with the power functions, were preserved under this assumption. Given that recurrent inhibition is prevalent in the neocortex (Kapfer et al., 2007; Shu et al., 2003; Silberberg and Markram, 2007), normalization of the activity in the location map is expected to occur at least to a certain extent (c.f., Carandini and Heeger, 1994; Hahnloser et al., 2000). Meanwhile, there may also exist feed-forward inhibition from the location map to the numerosity detector neurons. If the total activity of the location map increases with the numerosity, such feed-forward inhibition is expected to increase accordingly, thereby effectively increasing the threshold of the numerosity neurons. This is theoretically equivalent to decreasing each localized activity in the location map while keeping the threshold of the numerosity neurons constant, thereby possibly complementing the normalization within the location map.

As shown above, I have examined whether and how the model behavior changes according to the parameter values and the way the level of each localized activity decreases with the numerosity [as mentioned in the assumption (I)], showing that considerable flexibility is permitted for the main features to hold. It would be tempting to explore biologically detailed implementation of the proposed hypothetical mechanism. Examining whether and how single neuronal properties could implement branch-specific nonlinearity [assumption (III)] by using detailed neuron models has been an active research topic (Mel, 1993; Poirazi et al., 2003a,b; Rhodes, 2006), and the hypothesis that individual dendritic branches can operate as an independent functional unit has been extensively validated (Mel, 2007). Some of the important natures, such as the effect of inhibition applied onto branches (Jadi and Mel, 2007; Cosyne abstract) or the possibility of the existence of multiple functional compartments within a single branch (Major et al., 2008), however, are continuing to be actively studied. As for the branch-specific plasticity [assumption (II)], there are pioneering modeling studies (Mel, 1993; Poirazi and Mel, 2001). However, recent findings and indications such as the involvement of spatially restricted availability of the plasticity-related proteins (Govindarajan et al., 2006; Harvey and Svoboda, 2007; Harvey et al., 2008), branch-specific activity-dependent regulation of potassium channels (Losonczy et al., 2008), and activity-dependent local BDNF secretion (Tanaka et al., 2008), have not yet been considered in the model. Before that, whether cooperative plasticity induction among nearby dendritic sites actually occurs in the neocortical regions where numerosity detector neurons exist is expected to be clarified; so far cooperative plasticity induction has been demonstrated in the hippocampus and currently there is no evidence for or against in the neocortex. Effects of homeostatic plasticity (Ibata et al., 2008; Rabinowitch and Segev, 2006a,b, 2008; Turrigiano and Nelson, 2004) would also need to be considered. Construction of a realistic detailed model, incorporating all of these, is desired to be addressed in the future, although it is beyond the scope of the present paper. Nevertheless,
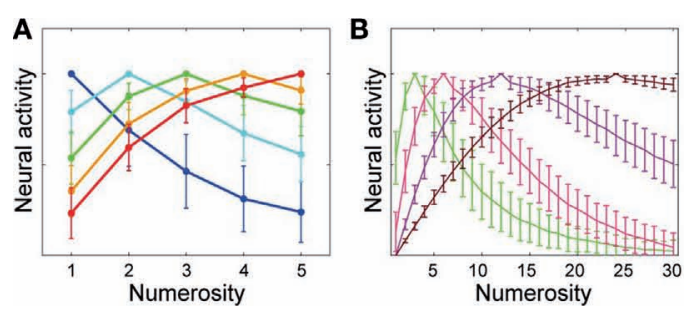
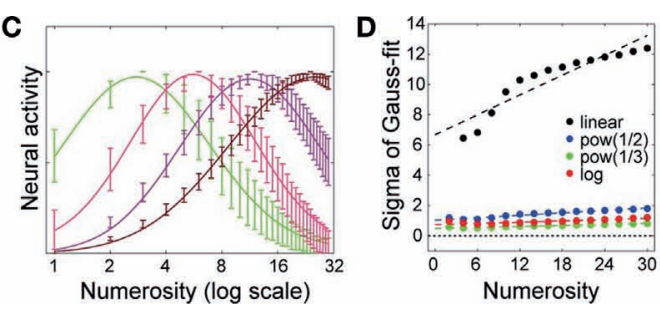

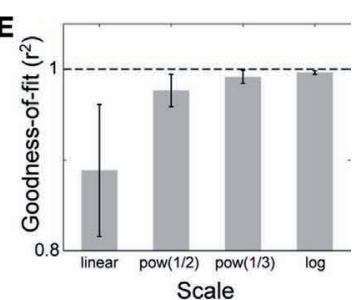

FIGURE 6 | Simulation results with a different way of normalization of the activity in the location map. Simulation results when the level of each localized activity in the location map decreases with the numerosity but the summation of them sublinearly increases; specifically, the sum of the squares of the localized activities was assumed to be constant. The main features of the model behavior were preserved under this assumption. Variability in the input magnitude and in the dendritic threshold was set to 0.25 (standard deviation per mean), and the convergence of multiple inputs corresponding to different portions of the location map onto the same branch was not considered. $(\mathbf{A}, \mathbf{B})$ Numerosity tunings in the cases of the preferred numerosity around $1 \sim 5$ (A) or 3, 6, 12, and 24 (B). (C) The same tuning curves in (B) was plotted on the logarithmic scale, with the Gaussian fitting curves. (D) Dependence of the tuning width (the standard deviation of the Gaussian fittings) on the preferred numerosity in four different scales. (E) Goodness of the Gaussian fit in the different scales. 
a previous study using a multicompartment model of pyramidal cell may support the plausibility of the mechanism proposed in this paper. Specifically, it was shown (Mel, 1993), in a different context from the number selectivity, that the firing activity of the pyramidal cell model was maximized when synaptic inputs are grouped into a certain size of (and thus a certain number of) spatially localized "clusters", and the "preferred number" (of clusters) varied according to conditions such as the type or the level of dendritic active conductance (see Fig. 7 of Mel, 1993).

\section{DISCUSSION \\ RELATIONSHIP WITH THE PREVIOUSLY PROPOSED HIERARCHICAL CIRCUITRY FOR NUMEROSITY TUNING}

I have proposed a hypothetical mechanism of how unimodal tuning for numerosity (Nieder and Merten, 2007; Nieder and Miller, 2004; Nieder et al., 2002) can be shaped through cooperative plasticity induction and nonlinear input integration at nearby dendritic sites, on the basis of the existing proposal (Dehaene and Changeux, 1993) that individual visual stimuli are represented as similar level of localized activities in a cortical area in the dorsal visual pathway. A previous modeling study (Verguts and Fias, 2004) demonstrated by simulation that the appropriate connection strengths between the summation clusters and the numerosity detector neurons (Figure 1A) in the hypothesized hierarchical circuitry (Dehaene and Changeux, 1993; Verguts and Fias, 2004) can be acquired by a biologically plausible unsupervised learning algorithm, if the summation clusters exist from the beginning of the simulation. However, they have not demonstrated that the connections from the location map to the summation clusters can also be formed via unsupervised learning; instead, they have shown (Verguts and Fias, 2004) that the summation clusters can be formed via supervised learning (backpropagation algorithm) if the numerosity detector neurons exist from the beginning. Therefore, so far it has remained unknown whether and how the unimodal tuning of the numerosity detector neurons is developed through plasticity mechanisms from the very beginning (i.e., without assuming that the summation clusters have already been shaped); for which the present study proposed a possible solution. Moreover, as shown above, the proposed model can explain a prominent feature of the numerosity detector neurons, namely, broadening of the tuning curve in proportion to the preferred numerosity (further discussed below). However, tuning curves obtained in the proposed model (Figure 4D, top, Figures $\mathbf{5 A}-\mathbf{F a}$ and $6 \mathrm{~A}$ ) were less sharp around the peaks than the reality (Nieder and Miller, 2004; Nieder et al., 2002), and thus the proposed mechanism may not operate by itself but rather cooperate with the previously proposed network-based mechanisms (Dehaene and Changeux, 1993; Verguts and Fias, 2004). Specifically, it seems possible that initially rough numerosity preference is shaped through the proposed mechanism, and the existence of such numerosity detector "precursor" neurons then facilitate subsequent network-level learning, resulting in generation of neurons having monotonic numerosity tuning (the summation clusters) and sharp unimodal tuning. So far, neurons showing unimodal numerosity tuning (Nieder and Merten, 2007; Nieder and Miller, 2003, 2004; Nieder et al., 2002) and those showing monotonic numerosity tuning (Roitman et al., 2007) were observed in different animals doing different tasks. It would be insightful if these two types of neurons are found in a single animal in the future.

\section{POSSIBLE DENDRITIC CONTRIBUTION TO THE WEBER-FECHNER LAW IN NUMEROSITY COMPARISON}

In the proposed mechanism, broadening of the tuning curve along with the preferred numerosity, which was experimentally observed and proposed to underlie the Weber-Fechner law-dependent accuracy in numerosity estimation and comparison, is naturally accompanied. Specifically, since the tuning curve width is exactly proportional to the preferred numerosity in the extreme case without variability in the input and the threshold (Figure 4A), the proportionality is expected to still roughly hold after incorporating the variability, as actually confirmed (Figure 4E). Therefore, the proposed model provides a possible mechanistic explanation of the Weber-Fechner law in numerical comparison. So far two psychological models have been proposed for the Weber-Fechner law, namely, logarithmically compressed representation of number (Dehaene and Changeux, 1993) and scale-free variability on the linear representation (Brannon et al., 2001; Gallistel and Gelman, 1992). They are rather similar, however, in their behavioral predictions (Dehaene, 2001), and distinguishable almost solely by the shape of the tuning curve; specifically, the shape should be symmetric on the log scale, i.e., asymmetric on the linear scale in the former but symmetric in the linear scale in the latter (Dehaene, 2003). The experimental results that the tuning curve of the numerosity detector neurons appeared most symmetric in the log scale (Nieder and Merten, 2007; Nieder and Miller, 2003) has been proposed to imply the logarithmic representation. Recent behavioral studies in humans (Dehaene et al., 2008) as well as in monkeys (Merten and Nieder, 2008) also support the compressed scaling. In the model presented in this paper, the tuning curve becomes most symmetric in the log scale, compared with the linear and power function scales (Figures 4D,F and 6B,C,E), if sufficient variability in the input and the threshold or the input convergence from different locations are incorporated (Figures $4 \mathrm{~B}$ and 5). It is therefore suggested that such a variability or input convergence could be a biological substrate of the compressed scaling of the mental number line, although whether and how they can be optimized so that a particular scaling, such as the logarithmic, gives the best symmetry is unclear.

\section{NUMEROSITY-TUNED PERSISTENT ACTIVITY}

Another important issue regarding the neural basis of numerical cognition is how the activity of some number-selective neurons can sustain after the disappearance of the stimuli with graded preferences to numerosities (Nieder and Miller, 2004; Nieder et al., 2002). Although it is very likely that excitatory reverberation plays a crucial role in the neuronal delay activity (Wang, 2001), sustaining a graded activity is not straightforward, since it corresponds to keeping a state at a neutrally, rather than an asymptotically, stable equilibrium (Brody et al., 2003; Machens et al., 2005; Miller et al., 2003). Interestingly, a previous modeling study (Goldman et al., 2003) has shown that if each dendritic branch of individual neurons in the network possesses bistability, i.e., capability of remaining at two different levels of activation (Loewenstein and Sompolinsky, 2003; Wang and Major, 2003), each neuron can show graded persistent activity 
depending on how many branches are in the activated state. It would be intriguing to construct a network model incorporating multiple dendritic branches of each neuron (Goldman et al., 2003; Morita, 2008) to see if such a model can explain numerosity-tuned transient as well as sustained neuronal activity, and moreover, computation utilizing such activity (Dehaene and Changeux, 1993).

\section{EXPERIMENTALLY TESTABLE PREDICTIONS}

In order to experimentally clarify if the mechanism proposed in this paper operates in the actual brain, possibly in combination with the previously proposed network-based mechanisms (Dehaene and Changeux, 1993; Verguts and Fias, 2004) as discussed above, it would be useful to examine whether the unimodal numerosity tuning is lost in the absence of inhibition by blocking GABAergic transmission in vivo, because inhibition onto the numerosity detector neurons seems essential in the network-based models (Dehaene and Changeux, 1993; Verguts and Fias, 2004) but it is not explicitly required in the proposed dendritic model. It would be probable, however, that the dendritic threshold considered in the proposed model is partly determined by the level of inhibition. In this case, the proposed model would predict that if inhibition is strengthened by applying GABA agonists and thus the threshold is increased, the peak of the tuning curve shifts downward, i.e., the neurons become to prefer smaller numerosities (c.f., Figure 3). In contrast, if only the network-based mechanism (Dehaene and Changeux, 1993; Verguts and Fias, 2004) operates, strengthening inhibition would reduce overall activity of the number-selective neurons but would not shift the peak position of the tuning curve (c.f., Figure 1A). Another existing model for unimodal numerosity tuning based on oscillations (Miller and Kenyon, 2007) assumes winner-takeall competition at the final stage, which could be implemented by lateral inhibition similar to what is considered in the network-based models (Dehaene and Changeux, 1993; Verguts and Fias, 2004), so that strengthening inhibition is expected not to shift the tuning curve; thus this is also expected to be distinguishable from the proposed model. In order to test the contribution of the proposed model, it would also be interesting to examine whether and how single neuronal properties such as the dendritic morphology or the spike width correlates with the numerosity preference in vivo. Examining the membrane property and the gene expression profile, as well as testing whether disturbing them affects the number selectivity, by in vivo patch-clamp experiments would also be desired.

\section{GENERAL DISCUSSION}

The notion that individual dendritic branches of neurons can operate as independent compartments in terms of both the function and the plasticity (Govindarajan et al., 2006; Koch et al., 1982; Mel, 1993; Poirazi and Mel, 2001; Poirazi et al., 2003b), developed in the theoretical research stream exploring the functional significance of dendrite (Ascoli, 2002; Koch, 1998; Koch and Segev, 2000; Koch et al., 1983; London and Häusser, 2005; Mainen and Sejnowski, 1996; Mel, 1994, 2007; Rall, 1964; Rinzel, 1975; Segev, 1995; Segev et al., 1995; Shepherd, 2003; Shepherd et al., 1985), has now acquired a lot of experimental supports (Gasparini et al., 2004; Harvey and Svoboda, 2007; Harvey et al., 2008; Larkum and Nevian, 2008; Losonczy and Magee, 2006; Losonczy et al., 2008; Milojkovic et al., 2005; Morita, 2009; Nevian et al., 2007; Polsky et al., 2004; Schiller et al., 2000; Wei et al., 2001). Not only suggested as a possible source of rich general computational power, dendritic compartmentalization of pyramidal cell has been proposed to play specific roles in particular cortical functions, such as translationinvariant orientation tuning (Mel et al., 1998) or binocular disparity (Archie and Mel, 2000). In the light of these latter works, the present study proposes a novel role of the compartmentalized dendrite in numerical cognition with novel insights into the issues raised therein, so as to postulate that single neuron property can directly contribute to abstract cognitive processes. The possibility that the same function can potentially be implemented either by a single neuron or by a network through similar self-organizing principles at two different scales (Morita, 2009) sounds redundant, and this may reflect evolutional processes that must have endowed biological systems with robustness. I would like to propose a more specific relationship between these two scales. Lower animals typically have a relatively small number of neurons each of which has a highly specialized function and thus is wired in a very specific way whereas higher animals possess much more neurons that would be less specialized and wired in a less specific manner. Given that ontogeny recapitulates phylogeny (Haeckel, 1866), however, it is conceivable that even in higher animals, individual neurons originally acquire highly specialized functions through single neuronal dendritic plasticity mechanisms in early stages of development, and such an individuality of single neurons will then facilitate the formation of functional circuits, which might otherwise be unrealistically difficult to be organized.

\section{DETAILED METHODS MODEL DETAILS}

The basic architecture of the model is described in the Section "Model", Figures 1B and 3. Neurons were assumed to have 50 dendritic branches in most simulations unless otherwise described. This value was chosen because it is in line with a study using a detailed model with a real morphology of the pyramidal cell (Poirazi et al., 2003b), which indicated that several dozen long thin terminal branches comprise independent nonlinear input integration subunits. I have confirmed that the main features of the model remain unchanged when 30 or 100 branches are assumed (see Figures 5E,F, respectively). In the simulations other than those for Figure 6, the magnitude of the input from a single localized activity in the location map to a single dendritic branch of the numerosity neuron was assumed to be distributed according to a normal distribution with the mean $\mu=1 / N$, where $N$ represents the numerosity of visual stimuli, and thus the summation of the inputs from all the localized activities was constant except for the variability. In the case of Figure 6, I assumed $\mu=1 / \sqrt{N}$, whereby the sum of the squares of the localized activities was constant except for the variability. The standard deviation per mean was set to 0 (i.e., no variation; Figures 3, 4A and 5B middle and bottom), 0.2 (Figure 5A), 0.25 (Figure 6), 0.4 (Figure 4B top and Figure 5C), or otherwise, 0.3. The dendritic threshold of individual neuron was assumed to be distributed according to a normal distribution. The standard deviation per mean was assumed to be 0 (i.e., no variation; Figures 3 and 4A,B top and bottom), 0.2 (Figure 5A), 0.25 (Figure 6), 0.4 (Figure 5C), or otherwise, 0.3 . The mean of the dendritic thresholds of individual neurons over branches was set to 0.3 (Figure 3A); 0.6 (Figure 3B); 0.36 (light blue), 
0.24 (green), and 0.15 (red) in Figures 4A,B. Regarding the large scale simulations (Figures 4D-F, 5 and 6), the mean was determined so that the number of neurons maximally tuned for each numerosity becomes roughly comparable. Specifically, approximate levels of the threshold to make a neuron tuned to the numerosities $1 \sim 30$ were at first estimated by a preparatory small-scale simulation. Then the relationship between the preferred numerosity and the threshold was fitted by a smooth function, as shown in black lines in Figures 7A,B for the cases where the summation of the localized activities, or the sum of their squares, was normalized, respectively. Thereafter, evenly spaced 3,000 points from 0.51 to 30.50 (i.e., 100 points centered at each of the tested numerosities $1 \sim 30$ ) were transformed by those functions, and the resulting values were used as an expected mean threshold value of 3,000 neurons, i.e., deviation obeying independent normal distribution was added to generate actual threshold value for each branch (see above). Red points and error-bars in Figures 7A,B indicate means and standard deviations of the individual neuron's mean threshold value (over branches) over neurons tuned for each numerosity in the simulations shown in Figures 4D-F and 6, respectively. Number of neurons maximally tuned for each numerosity is shown in Figures 7C,D; the number was comparable for any numerosity, as expected from the above procedure. Note that the relationship between the dendritic threshold and the preferred numerosity is rather steep for small numerosities [and it is steeper in the case where the summation of the localized activities was normalized (Figure 7A) than in the case where the sum of squares was normalized (Figure 7B)]. Therefore, if the threshold were assumed to be uniformly distributed over the same range, there must have emerged a gradient in the number of neurons tuned for each numerosity, i.e., there were expected to exist more neurons tuned for small numerosities than those tuned for large numerosities. It would be intriguing if this could be related to the experimental observation that there was an overrepresentation of neurons preferring small numerosities (Nieder and Merten, 2007), although there is little biological reason to assume that the dendritic threshold is uniformly distributed. In the analysis of the goodness of fit for the tuning curves (see below), average was first taken for each numerosity, before being averaged over numerosities, and thus, the number of neurons tuned for each numerosity does not have a direct effect. Input corresponding to each localized activity in the location map was randomly assigned onto a single dendritic branch without convergence (in Figures 3 and $4 \mathrm{~A}, \mathrm{~B}$ top and middle, Figures $5 \mathrm{~B}$ and 6 ) or with convergence of up to 3 times (Figures $4 \mathrm{~B}$ bottom, D-F and $\mathbf{5 A}, \mathbf{C}, \mathbf{E}, \mathbf{F}$ ) or up to 5 times (Figure 5D).

\section{SIMULATIONS AND ANALYSES}

Numerical simulations were performed by MATLAB (The Mathworks, Natick, MA, USA), using the built-in pseudo random number generating functions ("randn" and "randperm"). For each (simulated) neuron with a particular set of dendritic thresholds, 100 different sets of the input magnitudes for each of the numerosity $1 \sim 30$ were applied, and the mean activity of the neuron to each numerosity (over the input sets) was calculated to obtain the tuning curve. The tuning curve was then normalized, for each neuron, by setting the maximum (mean activity for the most preferred numerosity) as $100 \%$ and the minimum (mean activity for the least preferred numerosity) as $0 \%$, in the same way as done in the experimental studies (Nieder and Merten, 2007; Nieder and Miller, 2003). The normalized tuning curves for neurons having the same preferred numerosity, out of the 3,000 neurons (see above), were then averaged for each numerosity $(1 \sim 30)$; this "population average tuning curve" was plotted, with the standard deviation, in the figures (Figures $4 \mathrm{D}, \mathbf{5 A}-\mathrm{Fa}-\mathrm{c}$ and $\mathbf{6 A}-\mathrm{C}$ ). Gaussian functions were fitted to the population average tuning curves plotted in four different scales, namely, a linear scale, a power function with exponent of $1 / 2$, a power function with exponent of $1 / 3$, and a logarithmic scale, by minimizing the mean squared error according to the Nelder-Mead method by using "fminsearch" function of MATLAB. The goodness of fit was defined as $1-$ SSE/SST, where SST was the sum of squares total and SSE was the sum of squared errors. Since the fitting procedure was not appropriately converged for the numerosity 1 , or for 1 and 2 , in some cases, these date were not plotted in the figures nor used to calculate the linear regressions in Figure 4E top, Figures 5A-Fd and 6D (dashed lines) and the goodness of fit in Figures 4F, 5A-Fe and 6E. Specifically, data for the numerosity $2 \sim 30$ were plotted and used in the case of the
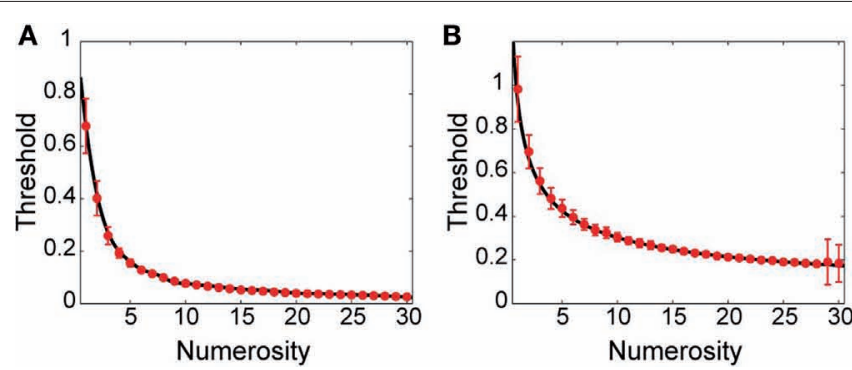

FIGURE 7 | Dendritic threshold and number of neurons tuned for each numerosity. $(\mathbf{A}, \mathbf{B})$ Dendritic threshold of neurons maximally tuned for each numerosity in the cases where the summation of the localized activities in the location map (A), or the sum of their squares (B), was normalized, respectively. Black lines indicate functions to fit the relationship between the preferred numerosities and the thresholds obtained from a preparatory small scale simulation. Evenly spaced 3,000 points from 0.51 to 30.50 were transformed by those functions, and the resulting values were used as an expected mean
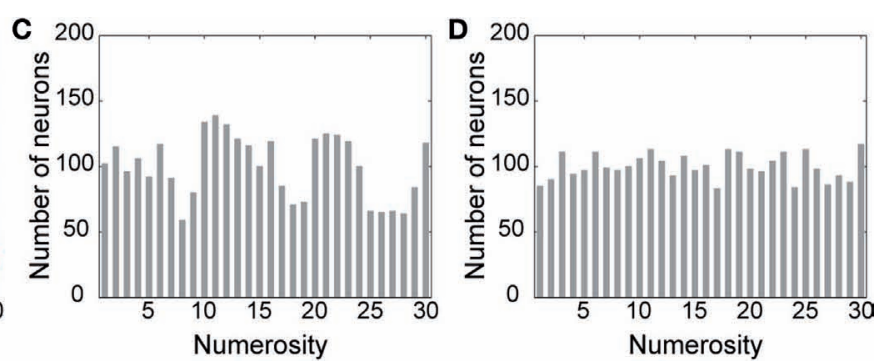

threshold value of 3,000 neurons (deviation obeying independent normal distribution was added to generate actual threshold value for each branch; see Model details). Red points and error-bars in (A) and (B) indicate means and standard deviations (over neurons) of the average threshold value (over branches) for neurons maximally tuned for each numerosity used in the simulations shown in Figures 4D-F and in Figure 6, respectively. (C,D) Number of neurons maximally tuned for each numerosity in the simulations shown in Figures 4D-F (C) and in Figure 6 (D) 
linear scale in Figures 4E,F and 5B,D,E and the case of the nonlinear scales in Figures 5C,F and 6; data for $3 \sim 30$ were plotted and used in the case of the linear scale in Figures 5C,F and 6; otherwise, all the data for $1 \sim 30$ were plotted and used.

\section{REFERENCES}

Amari, S. (1980). Topographic organization of nerve fields. Bull. Math. Biol. 42, 339-364.

Archie, K. A., and Mel, B. W. (2000). A model for intradendritic computation of binocular disparity. Nat. Neurosci. 3, 54-63.

Ascoli, G. A. (2002). Computational Neuroanatomy: Principles and Methods. Totowa, Humana Press.

Brannon, E. M., and Terrace, H. S. (1998). Ordering of the numerosities 1 to 9 by monkeys. Science 282, 746-749.

Brannon, E. M., Wusthoff, C. J., Gallistel, C. R., and Gibbon, J. (2001). Numerical subtraction in the pigeon: evidence for a linear subjective number scale. Psychol. Sci. 12, 238-243.

Brody, C. D., Romo, R., and Kepecs, A. (2003). Basic mechanisms for graded persistent activity: discrete attractors, continuous attractors, and dynamic representations. Curr. Opin. Neurobiol. 13, 204-211.

Carandini, M., and Heeger, D. J. (1994). Summation and division by neurons in primate visual cortex. Science 264, 1333-1336.

Dehaene, S. (1997). The Number Sense: How the Mind Creates Mathematics. New York, Oxford University Press.

Dehaene, S. (2001). Subtracting pigeons: logarithmic or linear? Psychol. Sci. 12, 244-246; discussion 247.

Dehaene, S. (2003). The neural basis of the Weber-Fechner law: a logarithmic mental number line. Trends Cogn. Sci. 7, 145-147.

Dehaene, S., and Changeux, J. P. (1993). Development of elementary numerical abilities - a neuronal model. J. Cogn. Neurosci. 5, 390-407.

Dehaene, S., Izard, V., Spelke, E., and Pica, P. (2008). Log or linear? Distinct intuitions of the number scale in Western and Amazonian indigene cultures. Science 320, 1217-1220.

Dehaene, S., Molko, N., Cohen, L., and Wilson, A. J. (2004). Arithmetic and the brain. Curr. Opin. Neurobiol. 14, 218-224.

Fechner, G. (1860). Elemente der Psychophysik. Leipzig, Breitkopf \& Härtel.

Gallistel, C. R., and Gelman, R. (1992). Preverbal and verbal counting and computation. Cognition 44, 43-74.
Gasparini, S., Migliore, M., and Magee, J. C. (2004). On the initiation and propagation of dendritic spikes in CA1 pyramidal neurons. J. Neurosci. 24, 11046-11056.

Golding, N.L., Staff, N.P., and Spruston, N. (2002). Dendritic spikes as a mechanism for cooperative long-term potentiation. Nature 418, 326-331.

Goldman, M. S., Levine, J. H., Major, G., Tank, D. W., and Seung, H. S. (2003). Robust persistent neural activity in a model integrator with multiple hysteretic dendrites per neuron. Cereb. Cortex 13, 1185-1195.

Govindarajan, A., Kelleher, R. J., and Tonegawa, S. (2006). A clustered plasticity model of long-term memory engrams. Nat. Rev. Neurosci. 7, 575-583.

Haeckel, E. (1866). Generelle Morphologie der Organismen. Berlin, G. Reimer.

Hahnloser, R. H., Sarpeshkar, R., Mahowald, M. A., Douglas, R. J., and Seung, H. S. (2000). Digital selection and analogue amplification coexist in a cortex-inspired silicon circuit. Nature 405, 947-951.

Harvey, C. D., and Svoboda, K. (2007). Locally dynamic synaptic learning rules in pyramidal neuron dendrites. Nature 450, 1195-1200.

Harvey, C. D., Yasuda, R., Zhong, H., and Svoboda, K. (2008). The Spread of Ras Activity Triggered by Activation of a Single Dendritic Spine. Science 321, 136-140. (2008). Rapid synaptic scaling induced by changes in postsynaptic firing. Neuron 57, 819-826.

Jadi, M., and Mel, B. W. (2007). Synchrony and location dependent effects of inhibition in a pyramidal neuron model. Computational and Systems Neuroscience Meeting Abstract. Poster II-91.

Kapfer, C., Glickfeld, L. L., Atallah, B.V., and Scanziani, M. (2007). Supralinear increase of recurrent inhibition during sparse activity in the somatosensory cortex. Nat. Neurosci. 10, 743-753.

Koch,C. (1998). Biophysics of Computation: Information Processing in Single Neurons. New York, Oxford University Press.

Koch, C., Poggio, T., and Torre, V. (1982). interpretation of dendritic morphology. Philos. Trans. R. Soc. Lond. B Biol. Sci. 298, 227-263.
Ibata, K., Sun, Q., and Turrigiano, G. G. Retinal ganglion cells: a functional

\section{ACKNOWLEDGMENTS}

This work was supported by Grant-in-Aid for Young Scientists (B) 19700310 from Ministry of Education, Culture, Sports, Science and Technology, Japan.

Koch, C., Poggio, T., and Torre, V. (1983). Nonlinear interactions in a dendritic tree: localization, timing, and role in information processing. Proc. Natl. Acad. Sci. U. S. A. 80, 2799-2802.

Koch, C., and Segev, I. (2000). The role of single neurons in information processing. Nat. Neurosci. 3(Suppl.), 1171-1177.

Kohonen, T. (1982). Self-organized formation of topologically correct feature maps. Biol. Cybern. 43, 59-69.

Larkum, M. E., and Nevian, T. (2008). Synaptic clustering by dendritic signalling mechanisms. Curr. Opin. Neurobiol. 18, 1-11.

Liu, G. (2004). Local structural balance and functional interaction of excitatory and inhibitory synapses in hippocampal dendrites. Nat. Neurosci. 7 , 373-379.

Loewenstein, Y., and Sompolinsky, H. (2003). Temporal integration by calcium dynamics in a model neuron. Nat. Neurosci. 6, 961-967.

London, M., and Häusser, M. (2005). Dendritic computation. Annu. Rev. Neurosci. 28, 503-532.

Losonczy, A., and Magee, J. C. (2006) Integrative properties of radial oblique Dendrites in hippocampal CAl pyramidal neurons. Neuron 50, 291-307.

Losonczy,A.,Makara,J.K.,andMagee, J. C. (2008). Compartmentalized dendritic plasticity and input feature storage in neurons. Nature 452, 436-441.

Machens, C. K., Romo, R., and Brody, C. D. (2005). Flexible control of mutual inhibition: a neural model of twointerval discrimination. Science 307, 1121-1124.

Mainen, Z. F., and Sejnowski, T. J. (1996). Influence of dendritic structure on firing pattern in model neocortical neurons. Nature 382, 363-366.

Major, G., Polsky, A., Denk, W., Schiller, J., and Tank, D. W. (2008). Spatiotemporally graded NMDA spike/plateau potentials in basal dendrites of neocortical pyramidal neurons. J. Neurophysiol. 99, 2584-2601.

Mehta, M. R. (2004). Cooperative LTP can map memory sequences on dendritic branches. Trends Neurosci. 27, 69-72.

Mel, B. W. (1993). Synaptic integration in an excitable dendritic tree. J. Neurophysiol. 70, 1086-1101.

Mel, B.W. (1994). Information processing in dendritic trees. Neural. Comput. 6 , 1031-1085.
Mel, B. W. (2007). Why have dendrites? A computational perspective. In Dendrites, 2nd edn, G. Stuart et al., eds (New York, Oxford University Press), pp. 421-440.

Mel, B. W., Ruderman, D. L., and Archie, K. A. (1998). Translationinvariant orientation tuning in visual "complex" cells could derive from intradendritic computations. J. Neurosci. 18, 4325-4334.

Merten, K., and Nieder, A. (2009). Compressed scaling of abstract numerosity representations in adult humans and monkeys. J. Cogn. Neurosci. 21, 333-346.

Miller, J. A., and Kenyon, G. T. (2007) Extracting number-selective responses from coherent oscillations in a computer model. Neural. Comput. 19, 1766-1797.

Miller, P., Brody, C. D., Romo, R., and Wang, X.-J. (2003). A recurrent network model of somatosensory parametric working memory in the prefrontal cortex. Cereb. Cortex 13, 1208-1218.

Milojkovic, B. A., Radojicic, M. S., and Antic, S. D. (2005). A strict correlation between dendritic and somatic plateau depolarizations in the rat prefrontal cortex pyramidal neurons. J. Neurosci. 25, 3940-3951.

Minsky, M., and Papert, S. (1969). Perceptrons: An Introduction to Computational Geometry. Cambridge, MA, The MIT Press. dritic compartmentalization in the spatial working memory circuit. J. Neurosci. 28, 7699-7724.

Morita, K. (2009). Computational implications of cooperative plasticity induction at nearby dendritic sites. Sci. Signal. 2, pe2.

Nevian, T., Larkum, M. E., Polsky, A., and Schiller, J. (2007). Properties of basal dendrites of layer 5 pyramidal neurons: a direct patch-clamp recording study. Nat. Neurosci. 10, 206-214.

Nieder, A. (2005). Counting on neurons: the neurobiology of numerical competence. Nat. Rev. Neurosci. 6, 177-190.

Nieder, A., and Dehaene, S. (2009). The representation of number in the brain.

Nieder,A., Freedman,D. J., and Miller, E. K. (2002). Representation of the quantity of visual items in the primate prefrontal cortex. Science 297, 1708-1711.

Nieder, A., and Merten, K. (2007). A labeled-line code for small and large
Morita, K. (2008). Possible role of denAnnu. Rev. Neurosci. 32, 185-208. 
numerosities in the monkey prefrontal cortex. J. Neurosci. 27, 5986-5993.

Nieder,A., and Miller, E. K. (2003).Coding of cognitive magnitude: compressed scaling of numerical information in the primate prefrontal cortex. Neuron 37, 149-157.

Nieder, A., and Miller, E. K. (2004). A parieto-frontal network for visual numerical information in the monkey. Proc. Natl. Acad. Sci. U. S. A. 101, 7457-7462.

Poirazi, P., Brannon, T., and Mel, B. W. (2003a). Arithmetic of subthreshold synaptic summation in a model CA1 pyramidal cell. Neuron 37, 977-987.

Poirazi, P., Brannon, T., and Mel, B. W. (2003b). Pyramidal neuron as two-layer neural network. Neuron 37, 989-999.

Poirazi, P., and Mel, B. W. (2001). Impact of active dendrites and structural plasticity on the memory capacity of neural tissue. Neuron 29, 779-796.

Polsky, A., Mel, B. W., and Schiller, J. (2004). Computational subunits in thin dendrites of pyramidal cells. Nat. Neurosci. 7, 621-627.

Rabinowitch, I., and Segev, I. (2006a). The endurance and selectivity of spatial patterns of long-term potentiation/ depression in dendrites under homeostatic synaptic plasticity. J. Neurosci. 26, 13474-13484

Rabinowitch, I., and Segev, I. (2006b). The interplay between homeostatic synaptic plasticity and functional dendritic compartments. J. Neurophysiol. 96 , 276-283.

Rabinowitch, I., and Segev, I. (2008). Two opposing plasticity mechanisms pulling a single synapse. Trends Neurosci. 31, 377-383.

Rall, W. (1964). Theoretical significance of dendritic trees for neuronal input-output relations. In Neural Theory and Modeling, R. F. Reiss, ed. (Stanford, CA, Stanford University Press), pp. 73-97.

Rhodes, P. (2006). The properties and implications of NMDA spikes in neocortical pyramidal cells. J. Neurosci. 26, 6704-6715.

Rinzel, J. (1975). Voltage transients in neuronal dendritic trees. Fed. Proc. 34, 1350-1356.

Roitman, J. D., Brannon, E. M., and Platt, M. L. (2007). Monotonic coding of numerosity in macaque lateral intraparietal area. PLoS Biol. 5, e208. doi: 10.1371/journal.pbio.0050208.

Sawamura, H., Shima, K., and Tanji, J. (2002). Numerical representation for action in the parietal cortex of the monkey. Nature 415, 918-922.

Schiller, J., Major, G., Koester, H. J., and Schiller, Y. (2000). NMDA spikes in basal dendrites of cortical pyramidal neurons. Nature 404, 285-289.

Segev, I. (1995). Dendritic processing. In The Handbook of Brain Theory and Neuronal Networks, M. Arbib, ed. (Cambridge, MA, The MIT Press), pp. 282-289.

Segev, I., Rinzel, J., and Shepherd, G. M. (1995). The Theoretical Foundation of Dendritic Function: Selected Papers of Wilfrid Rall with Commentaries. Cambridge, MA, The MIT Press.

Shepherd, G. M. (2003). Information processing in complex dendrites. In From Molecules to Networks: An
Introduction to Cellular and Molecular Neuroscience, J. Byrne and J. Roberts, eds (San Diego, CA, Academic Press), pp. 479-497.

Shepherd, G., Brayton, R., Miller, J. Segev, I., Rinzel, J., and Rall, W. (1985). Signal enhancement in distal cortical dendrites by means of interactions between active dendritic spines. Proc. Natl. Acad. Sci. U. S. A. 82, 2192-2195.

Shu, Y., Hasenstaub, A., and McCormick, D. A. (2003). Turning on and off recurrent balanced cortical activity. Nature 423, 288-293.

Silberberg, G., Markram, H. (2007) Disynaptic inhibition between neocortical pyramidal cells mediated by Martinotti cells. Neuron 53, 735-746.

Sjöström, P. J., Rancz, E. A., Roth, A., and Häusser, M. (2008). Dendritic excitability and synaptic plasticity. Physiol. Rev. 88, 769-840.

Tanaka, J., Horiike, Y., Matsuzaki, M., Miyazaki, T., Ellis-Davies, G. C., and Kasai, H. (2008). Protein synthesis and neurotrophin-dependent structural plasticity of single dendritic spines. Science 319, 1683-1687.

Turrigiano, G. G., and Nelson, S. B. (2004) Homeostatic plasticity in the developing nervous system. Nat. Rev. Neurosii. 5, 97-107.

Verguts, T., and Fias, W. (2004). Representation of number in animals and humans: a neural model. J. Cogn Neurosci. 16, 1493-1504.

von der Malsburg, C. (1973). Self-organization of orientation sensitive cells in the striate cortex. Kybernetik 14, 85-100.
Wang, S. S., and Major, G. (2003). Integrating over time with dendritic wave-fronts. Nat. Neurosci. 6, 906-908.

Wang,X.-J.(2001).Synaptic reverberation underlying mnemonic persistent activity. Trends Neurosci. 24, 455-463.

Weber, E. (1850). Der Tastsinn und das Gemeingefühl. In Handwörterbuch der Physiologie, Vol. 3, Part 2, R. Wagner, ed. BraunschweigVieweg), pp. 481-588.

Wei, D. S., Mei, Y. A., Bagal, A., Kao, J. P., Thompson, S. M., and Tang, C. M (2001). Compartmentalized and binary behavior of terminal dendrites in hippocampal pyramidal neurons. Science 293, 2272-2275.

Conflict of Interest Statement: The author declares that the research was conducted in the absence of any commercial or financial relationships that could be construed as a potential conflict of interest.

Received: 31 March 2009; paper pending published: 26 July 2009; accepted: 26 July 2009; published online: 10 August 2009

Citation: Morita K (2009) Possible dendritic contribution to unimodal numerosity tuning and Weber-Fechner law-dependent numerical cognition. Front. Comput. Neurosci. 3:12. doi: 10.3389/neuro.10.012.2009 Copyright () 2009 Morita. This is an openaccess article subject to an exclusive license agreement between the authors and the Frontiers Research Foundation, which permits unrestricted use, distribution, and reproduction in any medium, provided the original authors and source are credited. 